\title{
Building up an Internet of Things Based on the Mapping Between EPC and IPv6
}

\author{
C. DENG, H. DENG, and X. CHEN
}

\begin{abstract}
In view of the similarity between the EPC (Electronic Product Code) and IPv6 addresses in the logic hierarchical structure and after deep analysis of EPC and IPv6 address, this paper puts forward a mapping mechanism between EPC and IPv6 address. The address mapping structure between IPv6 address and EPC code makes the locating function of IPv6 address and the identifying function of EPC combined. After that routers can locate items via the information on network prefix and the information on EPC is used to identify the object, so that when querying item's detailed information, it turns to locate the EPCIS (EPC Information Services) server where the item is stored just using RFID-IPv6 gateway, without relying on the ONS (Object Naming Service) complex analytic process.
\end{abstract}

Keywords - Electronic Product Code (EPC), EPC Information Services (EPCIS), Internet Protocol version 6 (IPv6), Internet of Things (IoT), Radio Frequency Identification (RFID)

\section{INTRODUCTION}

The advent of RFID (Radio Frequency Identification) technology and the coming-in-use Internet protocol IPv6 technology makes it possible for people to build Internet of Things that any item information can be accessed at anytime and anywhere, for RFID can provide each item in the real world with each ID, and IPv6 is able to provide the corresponding IP address.

But to build Internet of Things [1] based on the combination of RFID technology and IPv6 network structures, we need to study the mapping characteristics between electronic product code (EPC) and IPv6 address, as well as how to build "Internet of Things" based on them.

\section{II.COMPATIBILITY OF EPC CODE WITH IPv6 ADDRESSES}

\section{A. Hierarchical Topology of EPC}

EPC is a new coding standard of new generation of coding that is compatible with EAN / UPC. In the EPC system EPC combines with GTIN, therefore, EPC is not to replace the existing bar code standards, but the latter will gradually transit to the former or in the future supply chain, EPC and the EAN.UCC system will coexist.

There are three EPC coding schemes: EPC-64, EPC-96 and EPC-256. As shown in TABLE I, the commonly used is the

C. DENG is with the Guangzhou College of South China University of Technology, Guangzhou, 510800, China .

H. DENG and X. CHEN are with South China University of Technology, Guangzhou,510006, China
EPC-64 coding scheme, but EPC tags of new generation will adopt EPC-96 coding scheme. In a short time, EPC standards will not completely replace the existing coding standards, but keep compatibility with the other mainstream coding systems (GID, GTIN, SSCC, etc.)

\begin{tabular}{|c|c|c|c|c|c|}
\hline \multicolumn{7}{c|}{ TABLE I: EPC CODING STRUCTURE [2] } \\
\hline \multirow{3}{*}{ System } & Class & $\begin{array}{c}\text { Ver } \\
\text { No. }\end{array}$ & $\begin{array}{c}\text { Domain name } \\
\text { management }\end{array}$ & $\begin{array}{c}\text { Object of } \\
\text { classification }\end{array}$ & $\begin{array}{c}\text { Serial } \\
\text { No. }\end{array}$ \\
\hline \multirow{3}{*}{ EPC-64 } & Class I & 2 & 21 & 17 & 24 \\
\cline { 2 - 7 } & Class II & 2 & 15 & 13 & 24 \\
\cline { 2 - 7 } & Class III & 2 & 16 & 13 & 23 \\
\hline EPC-96 & Class I & 8 & 28 & 24 & 36 \\
\hline \multirow{3}{*}{ EPC-256 } & Class I & 8 & 32 & 56 & 192 \\
\cline { 2 - 7 } & Class II & 8 & 64 & 56 & 128 \\
\cline { 2 - 7 } & Class III & 8 & 128 & 56 & 64 \\
\hline
\end{tabular}

The EPC namespace is divided into four parts: the version number, domain name management, object classification and serial number. This method of partition provides EPC namespace with a four-layer tree topology: layer0 - Root, layer1- version number, layer2 - the domain name management, layer3 - storey object classification and layer4 - serial number. Each node in the namespace topology is marked by a binary number with 0 (the total digit of the number determined by the version number). In the same layer different node has a different label, but in different layers, different node may have the same label. Label zero is reserved as the root of the topology tree.

The path from the root node to a leaf node corresponds to a valid EPC. EPC value is determined by the code connecting the root node to a leaf node. The difference in EPC codes only occur in their version number, i.e., their domain name management, object classification and serial number are identical except for the zero-node. Moreover, the codes for three layers are defined identically so that they correspond to the same physical entity. Therefore, in fact, EPC coding namespace consists of three levels of hierarchy. One item can be allocated with several EPC codes, and once an EPC code is allocated, it will always be linked to the object.

\section{B. Hierarchical Topology of IPv6}

An IPv6 address is a 128-bit identifier. There are three types of IPv6 addresses: unicast address, multicast address and anycast address [3]. IPv6 address provides a globally unique identifier for the network interface. In this paper, the IPv6 address is divided into three parts: the public topology, site topology and the interface ID. This division makes IPv6 namespace have an outward limited tree topology, in which 
there are at least three layers: Layer0 - root node, layer 1 - public topology, layer2 - site topology, layer3 - interface identifier. There may be additional division in the public topology identifier, site topology and interfaces identifier. The formatted prefix (equivalent to 001 in binary) corresponds to the most important three bits in the public topology identifier. Each node in the namespace topology has identifier of a binary pattern. The identifier of the public topology is 48-bit; the one of site topology is 16-bit, and the one for the interface is 64-bit. The nodes at the same level may not have the same label, but the nodes at different level may have. Label-zero is reserved for the root in the tree topology.

The path from the root node to a leaf node corresponds to a valid IPv6 address. IPv6 address is determined by the node labels on the path from the root to the leaf node.

\section{Comparison and Compatibility Analysis}

The EPC-coding scheme and the IPv6 address scheme are designed to identify items. EPC-coding scheme is designed to uniquely identify all physical objects/item, and the IPv6 address scheme is designed to identify the network interface of a particular object/item. An EPC-code does not contain object's information related to it or information beyond the instructions of how-to-use it. EPC-code does not intend to embed the additional information. Therefore, EPC-coding scheme and the IPv6 address scheme cannot replace with each other.

The IPv6 addressing scheme and the EPC-coding scheme are very similar; TABLE II compares the IPv6 address with the main properties of EPC-coding.

TABLE II: COMPARISON OF EPC AND IPV6

\begin{tabular}{|c|c|c|}
\hline \multicolumn{3}{|c|}{ Comparison of EPC with IPv6 and compatibility analysis } \\
\hline Attributes & EPC & IPv6 \\
\hline Object recognition & All objects & Network interface \\
\hline Main application & Information indicators & Routing address \\
\hline Identifying area & Global & Global \\
\hline Unique identification & Unique & Unique \\
\hline $\begin{array}{c}\text { Same identifier is allocated to } \\
\text { multiple objects }\end{array}$ & No & Yes \\
\hline $\begin{array}{c}\text { Whether the identifier is } \\
\text { permanently allocated }\end{array}$ & Yes & No \\
\hline ID code & Binary & Binary \\
\hline ID length & $64,96,256$ & 128 \\
\hline Maximum depth of namespaces & 3 & 3 \\
\hline Assigner & Manufacturers & $\begin{array}{l}\text { Network } \\
\text { administrator }\end{array}$ \\
\hline
\end{tabular}

The IPv6 addressing namespace contains the three-layer structure for the purpose of distribution that EPC namespace, in fact, also contains. Both structures have designed the effective allocation and management structure for their respective namespace.

Nevertheless, these two are designed to meet the different needs of applications. Thus, the delimitation symbols of the two identification proposals have different performance. The partition in EPC effectively identifies the manufacturer of the items, product categories (determined by the manufacturer) and the unique product monomer. The partition in IPv6 addresses effectively identifies the network that a net interface belongs to or the partition of subnet. This partition has been used as the gateway address (or path and direction) by communication / routing system network interface. Due to their different expressions, EPC namespace is effectively managed by manufacturers and IPv6 namespace by network administrator.

\section{MAPPING BETWEEN EPC CODING AND IPv6 ADDRESSING}

An identifier must have two aspects of the function: First, to identify an object or product/item in the real world. For example, through EPC embedded in the real world, the network can acquire the identity and attributes of object or product through EPC; Second, through IPv6, to uniquely locate entities address in the network as an address. Only if having the address, can entities communicate with each other by addresses in the net.

By the discussion as before, we can see that EPC-coding scheme is designed to uniquely identify all physical objects, and the IPv6 address of the program is designed to identify the network interface of a particular object. Though the structure of the namespace in these two schemes is very similar, they cannot replace with each other. So, it is indispensable to combine them together.

In this section, we attempt to develop a key approach to constructing an Internet of Things and achieve end-to-end communications between entities through combining the IPv6 address and EPC identifier code. Because the IPv6 address cannot replace the product ID and their respective function is mutually indispensable to the construction of Internet of Things, so how to combine IPv6 address and the identifier is the key issue. Since EPC is widely used, in this paper, we use the mapping between EPC-coding and IPv6 addressing as an example to elaborate the key approach.

\section{A. IPv6 interface ID}

As shown in Fig. 1, IPv6 addresses can be divided into two parts of the same length: the first 64-bit for the subnet prefix, the next 64-bit is for the interface ID.

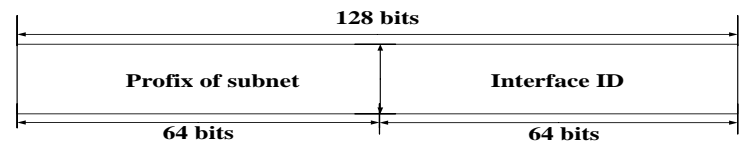

Fig. 1. Basic structure of IPv6 address

Subnet prefix is the basis for the router to locate, and the interface ID is the unique identifier of the device in subnet. IPv6 interface ID are as follows [4]:

1) The 64-bit interface ID derived from the address of the Extended Unique Identifier (EUI-64);

2 ) interface ID randomly generated that changes over time in order to provide a certain degree of crypticity;

3 ) the interface ID distributed in address auto-configuration process of the state.

RFC3513 declares [5] that all unicast address whose prefix is between 001-111 must use a 64-bit interface ID derived from EUI-64. EUI-64 address can be an address allocated to the network adapter, and also can be a derivative from IEEE802 (MAC address) addresses. Its derivative process is as shown in Fig. 2. 


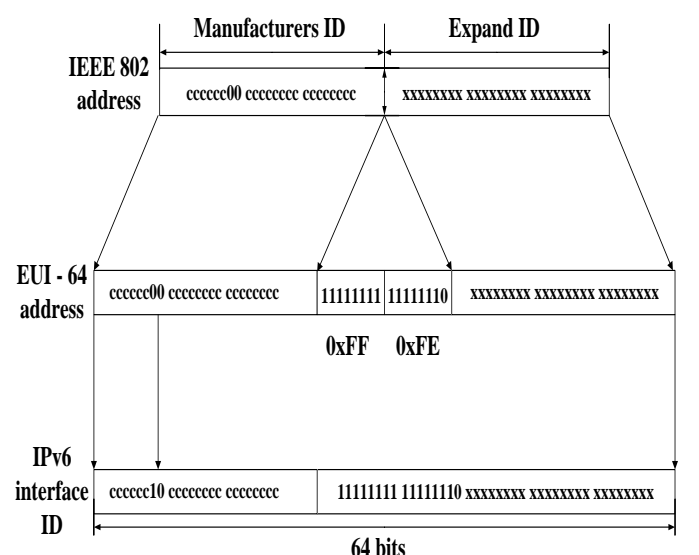

Fig. 2. Mapping among IEEE802 address, EUI-64 address and the IPv6 interface ID

\section{B. Mapping between EPC and IPv6}

Through the mapping relations among the IEEE 802 address, EUI-64 address and the IPv6 interface ID, we now put forward the mapping between EPC and IPv6. Taking EPC-64 and EPC-96 as an example, as shown in Fig. 3, we can see how IPv6 address is mapped to EPC-code.

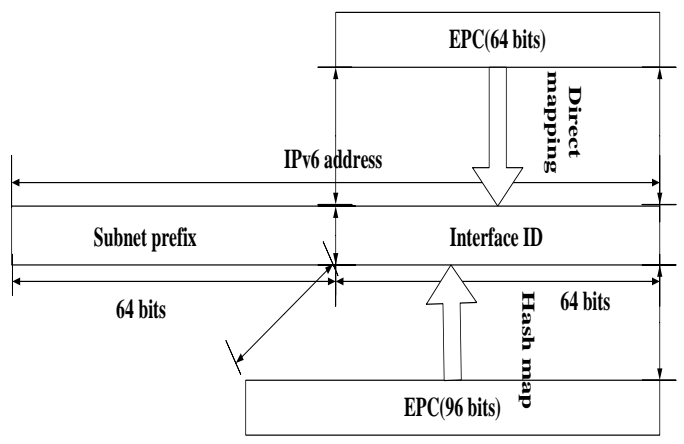

Fig. 3 Mapping between IPv6 and EPC-code

In this mapping, we can make the objects classification and serial number in EPC map to interface ID in IPv6 address. The new mapping structure of IPv6 address contains the EPC coding information, which makes the entity having a new mapping structure of IPv6 address may communicate with the devices having IPv6 addresses.

\section{RFID-IPv6 GATEWAY}

Gateway [6] is a gate connecting one network to another network. In the Internet network, the gateway is an intermediate device connecting the internal network and other network in the Internet, also known as "routers." Gateway address can be understood as the address of the channel used to transmit information from the internal network to the Internet.

To enable EPC network and the next generation of IPv6-based networks to communicate with each other, we must connect the two networks with the gateway. In this paper we try to use RFID-IPv6 gateway to connect the two networks. As Fig. 4 shows, RFID-IPv6 gateway is the connector designed to connect EPC network and IPv6 network. In the net layer, each EPC network uses RFID-IPv6 gateway to connect to the IPv6 network. While management of the Gateway is mainly the gateway IP address settings, where, using the new IPv6 address generated by the EPC-IPv6 mapping mechanism as IP address of RFID-IPv6 gateway, we can achieve our goal that the EPC network and IPv6 network communicate with each other.

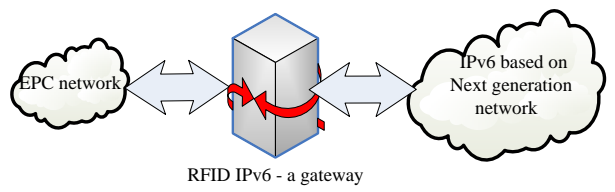

Fig. 4. RFID-IPv6 gateway

\section{V.QUERY PROCESS AFTER MAPPING}

In the system of Internet of Things, EPC-code is a strict and precise item identifier, detailed item information recorded in the EPCIS (EPC Information service) [7]. If wanting to know the details of items, after the reader to obtain the EPC-code of goods, one should upload the EPC-code to the middleware of information network system through the Internet or other means of communication, and then obtain the object name of products through the ONS (Object Name Service) [8] analysis, and then get a variety of services related product information through a variety of EPCIS interfaces.

The EPC-IPv6 mapping mechanism in this paper makes the post-mapped IPv6 address structure, not only own the original identifying function of the network address, but also have the identifying feature of items. So, to query the details about items, without through the complex analytical process of ONS, just with the RFID-IPv6 gateway, you can easily position EPCIS server corresponding to items.

As Fig. 5 shows, in order to be compatible with the existing net system of EPCglobal [9] Internet of Things, in this paper we not only design a query process to query the EPCIS server through the EPC-coding, but also a query process to query the EPCIS through the mapping mechanism of EPC-IPv6.

EPC - IPv6 mapping mechanism query process

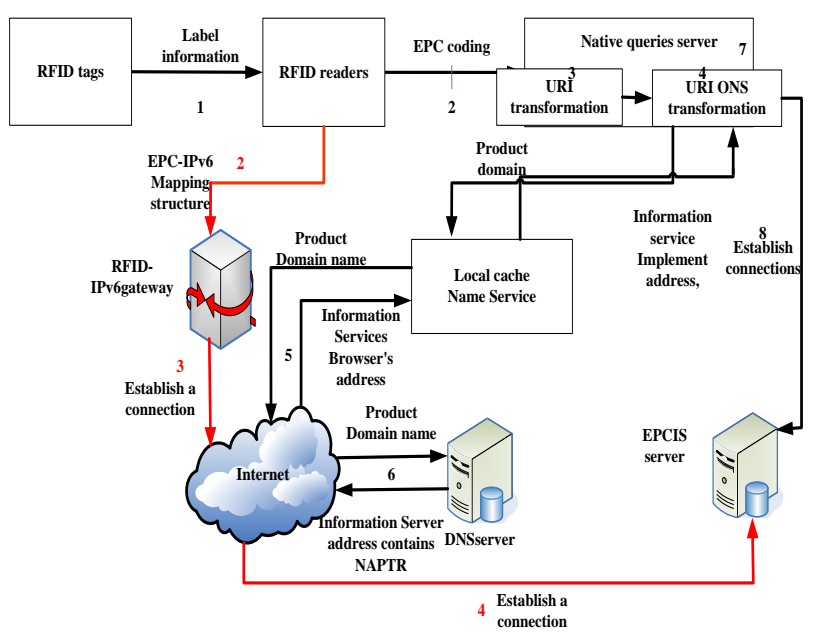

Fig. 5. Query process of mapping mechanism of EPC-IPv6

Detailed steps of inquiries are as follows:

1) RFID reader to read binary code in RFID electronic tags. 
2) RFID reader to resolve the binary code information; if the code has the structure of the EPC-IPv6 mapping, the it will be sent to the RFID-IPv6 gateway, and then the reader directly connects to goal EPCIS server through the RFID-IPv6 gateway; if it is EPC-code, then it is sent to the local query server.

3) Local query server receives EPC-code, transforms it into the format that complies with the EPCglobal tag data standard, and send it to ONS solver.

4) ONS solver transforms the data complying with the EPCglobal tag data standard format into the domain name format.

5) The local system sends query of DNS to the DNS (Domain Name Service) [10] servers by the Internet with a view to obtaining the corresponding NAPTR (Naming Authority Pointer) [11] record.

6) DNS server returns the NAPTR record containing service information.

7) ONS solver extracts NAPTR records from the server address and sends it to the local query server.

8) Local query server interacts with the EPCIS server containing its address to obtain the item information corresponding to the label.

\section{INFORMATION FORMAT BASED ON EPC-IPv6 MAPPING MECHANISM}

The data collected by RFID readers exhibit different formats defined by different factories. However, the data the computer can handle must follow certain standard. In the Internet of Things, the developers following such a standard can operate the RFID equipment using computer in a programmable way. Fig. 6 shows the general format of RFID tag data.

\begin{tabular}{|l|l|l|}
\hline RFID type & Data type & Reserved bits \\
\hline \multicolumn{3}{|c|}{ EPCcode(96 bits) } \\
\hline \multicolumn{3}{|c|}{ Information data } \\
\hline
\end{tabular}

Fig. 6. General format of RFID Data

As shown in Fig. 7, in the network, RFID tag data can be carried through TCP payload in order to achieve reliable transmission.

\begin{tabular}{|c|c|c|}
\hline $\begin{array}{c}\text { IPv6 } \\
\text { Basic header }\end{array}$ & $\begin{array}{c}\text { TCP } \\
\text { header }\end{array}$ & RFID data \\
\hline
\end{tabular}

Fig.7. IPv6, RFID and TCP Payload

Considering the advantages of IPv6, RFID information can also use extensible IPv6 header instead of effective load. This method can make the network layer exchange information more effectively. Fig. 8 shows the message format of RFID in the IPv6 extensible header.

\begin{tabular}{|c|c|c|c|}
\hline \multirow{2}{*}{\multicolumn{2}{|c|}{$\begin{array}{c}\text { Communication types } \\
\text { Payload length }\end{array}$}} & \multicolumn{2}{|c|}{ Flow Label } \\
\hline & & Next header & Hop limit \\
\hline \multicolumn{4}{|c|}{ Source address (128) } \\
\hline \multicolumn{4}{|c|}{ Destination address (128) } \\
\hline Next header & Header length & I Optional type & Optional length \\
\hline RFID type & Data type & \multicolumn{2}{|c|}{ Reserved bit } \\
\hline \multicolumn{4}{|c|}{ EPC code (96) } \\
\hline \multicolumn{4}{|c|}{ Data } \\
\hline
\end{tabular}

Fig. 8. Message format of RFID in the IPv6 extensible head

\section{CONCLUSIONS}

Based on the deep analysis and detailed comparison of EPC-coding and the architecture of IPv6 addresses, we proposed an address structure of mapping EPC-code to IPv6 address, which integrates the locating functions of IPv6 addresses and the identifying functions of EPC-code. Hence while querying the details of items, without needing the complex analytical process of ONS, just needing the RFID-IPv6 gateway, we can easily position the EPCIS server items correspond. In order to make RFID information transmittable in the network, we suggested taking use of the TCP payload and the expandable IPv6 header to carry RFID information.

\section{REFERENCES}

[1] ITU Internet Reports 2005: The Internet of Things [Online], 7th edition, http://handle.itu.int/11.1002/pub/800eae6f-en

[2] Guozhu Liu and Chunnian Ren, EPC Internet of Things Technology, Xidian University Press, Oct.2016

[3] Pete Loshin, IPv6 Clearly Explained, Elsevier Science \& Technology Books, 1999.

[4] IPv6 Interface Identifiers [Online], https://msdn.microsoft.com/en-us/library/aa915616.aspx

[5] Internet Protocol Version 6 (IPv6) Addressing Architecture [Online], https://tools.ietf.org/html/rfc3513

[6] Chonggao Yao, "Design and research of home gateway based on IPv6", Master Thesis, Guizhou University, Guiyang. China, June of 2007

[7] EPCIS[Online], https://en.wikipedia.org/wiki/EPCIS

[8] EPCglobal Object Name Service [Online], https://en.wikipedia.org/wiki/Object_Naming_Service

[9] EPCglobal [Online], https://en.wikipedia.org/wiki/EPCglobal

[10] Domain names - concepts and facilities [Online], https://tools.ietf.org/html/rfc1034

The Naming Authority Pointer (NAPTR) DNS Resource Record [Online], https://tools.ietf.org/html/rfc2915 